\title{
UM PANORAMA SOBRE TEORIAS E TÉCNICAS DE INCUBAÇÃO DE SONHOS
}

\author{
Vanessa Oliveira GOMES \\ Lenny Francis Campos DE ALVARENGA ${ }^{2}$ \\ Claudio Herbert NINA-E-SILVA ${ }^{2}$
}

${ }^{1}$ Acadêmica de Psicologia / Universidade de Rio Verde (UniRV).
${ }^{2}$ Professor Adjunto, Laboratório de Psicologia Anomalística e Neurociências / UniRV.

Recebido em: 19/09/2015 - Aprovado em: 18/07/2016 - Disponibilizado em: 18/12/2016

RESUMO: A incubação de sonhos diz respeito a uma série de técnicas de direcionamento da atenção a questões específicas antes de dormir com o objetivo de que os processos cognitivos e afetivos que ocorrem durante os sonhos possibilitem o autoconhecimento, a solução de problemas e/ou o desenvolvimento da criatividade. Este artigo teve como objetivo elaborar um panorama sobre as teorias e métodos de incubação de sonhos. Para tanto, foram apresentadas as teorias explicativas mais relevantes e as técnicas principais para a realização da incubação de sonhos por qualquer pessoa que se interesse pelo fenômeno. A última parte do artigo pretendeu ser um guia passo-a-passo de como conseguir realizar a incubação de sonhos.

Palavras-chave: Sonhos. Incubação de Sonhos. Psicologia Analítica. Psicanálise. Neurofisiologia.

ABSTRACT: The concept of dream incubation is related to a series of focusing attention techniques that intend to employ the cognitive and affective processes that occur during dreams to allow self-knowledge, problem solving and/or development of creativity. This study aimed to develop a panorama of the theories and methods of dream incubation. Thus, we present the most relevant explanatory theories and key techniques for the realization of dream incubation by anyone who is interested in this phenomenon. The last part of the article intended to be a step-by-step guide on how to accomplish the dream incubation.

Keywords: Dreams. Dream Incubation. Analytical Psychology. Psychoanalysis. Neurophysiology.

\section{Introdução}

Este artigo é uma pesquisa bibliográfica de caráter teórico descritivo que visou a apresentar ao público interessado o tema da incubação de sonhos, suas possíveis utilizações e as técnicas mais conhecidas utilizadas para se alcançar o fenômeno.

A incubação de sonhos diz respeito a uma série de técnicas de direcionamento da atenção a questões específicas antes de dormir com o objetivo de que os processos cognitivos e afetivos que ocorrem durante os sonhos possibilitem a solução de problemas e/ou o desenvolvimento da criatividade (WHITE;
Há vários estudos que evidenciaram a importância dos sonhos para o desenvolvimento da criatividade e para a solução efetiva de problemas nos campos científico, artístico e psicoterápico (LABERGE; RHEINGOLD, 1990; BARRETT, 2001; HILL, 2004; SCHREDER; ERLACHER, 2007; LABERGE; GAKENBACH, 2013).

Entretanto, variáveis tais como a frequência de recordação de sonhos e traços de personalidade parecem influenciar diretamente na capacidade de uso dos sonhos para a resolução de problemas e o TAYTROE, 2003). 
desenvolvimento

da

criatividade

(SCHREDER; ERLACHER, 2007).

Do ponto de vista psicológico, os sonhos são parte do mecanismo de compensação que regula a psique (JUNG, 1964/2008a; JUNG, 1964/2008b; HALL, 1997). Nesse sentido, "a função geral dos sonhos é tentar reestabelecer a nossa balança psicológica, produzindo um material onírico que reconstitui, de maneira sutil, o equilíbrio psíquico total" (JUNG, 1964/2008b, p.56).

De acordo com Hall (1997, p.31-32), a atividade compensatória dos sonhos pode ocorrer de três formas distintas:

\begin{abstract}
"Em primeiro lugar, o sonho pode compensar distorções temporárias na estrutura do ego, dirigindo o indivíduo a um entendimento mais abrangente das atitudes e ações. (...) Um segundo e mais profundo modo de compensação é aquele em que o sonho, como auto-representação da psique pode colocar uma estrutura do ego em funcionamento face-a-face com a necessidade de uma adaptação mais rigorosa ao processo de individuação. (...) Tornou-se cada vez mais claro para mim, entretanto, que existe um terceiro processo mais misterioso e sutil (...). O sonho pode ser visto como uma tentativa de alterar diretamente a estrutura de complexos sobre os quais o ego arquetípico se apóia, para a identidade em níveis mais conscientes".
\end{abstract}

Por outro lado, na perspectiva neurofisiológica, os trabalhos de Dement (1966/1978) sobre a psicofisiologia dos sonhos e os estudos de LaBerge (1985) e LaBerge e Rheingold (1990) sobre as bases neurobiológicas dos sonhos lúcidos demonstraram correlações fisiológicas importantes entre o que ocorre em nossos sonhos e em nosso corpo e vice-versa. Dessa maneira, esses autores evidenciaram que alterações neurofisiológicas induzidas por exercícios específicos podem influenciar no conteúdo dos sonhos, ressaltando a base biológica da incubação de sonhos.

O trabalho pioneiro no estudo científico dos sonhos no século XIX foi "A Interpretação dos Sonhos" (FREUD, 1899/2010). No primeiro capítulo desse livro, Freud descreveu uma exaustiva revisão da literatura sobre os sonhos até 1899 e relatou os estudos que demonstravam a relação entre os sonhos, a memória dos sonhos e a vida de. Já no capítulo 5 de “A Interpretação dos Sonhos", Freud (1899/2010, p.174) descreveu a influência de material recente no conteúdo dos sonhos e sintetizou as suas observações na afirmação de que “(...) em todo sonho, é possivel encontrar um ponto de contato com as experiências do dia anterior". Desse modo:

\footnotetext{
"Sempre que se afigura, a princípio, que a fonte de um sonho foi uma impressão de dois ou três dias antes, a pesquisa mais detida tem-me convencido de que a impressão foi lembrada na véspera, e assim tem sido possível demonstrar que uma reprodução da impressão, ocorrida no dia precedente, poderia ser inserida entre o dia do acontecimento original e o momento do sonho; além disso, tem sido possível indicar a eventualidade do dia anterior que teria levado à lembrança da impressão mais antiga" (FREUD, 1899/2010, p.175).
}

Esse trecho de "A Interpretação dos Sonhos" sobre a influência do material recente no conteúdo dos sonhos é importante para fundamentar a técnica de incubar os sonhos, visto que essa técnica depende justamente do material recente descrito por Freud (1899/2010). 


\section{O conteúdo dos sonhos}

Em "A Relação dos Sonhos com a Vida de Vigília", Freud (1899/2010) afirma que sonhar significa desligar-se do mundo real, mas estar ao mesmo tempo embarcado em nossas realidades mais relevantes $\mathrm{e}$ significativas. Nessa concepção, os sonhos são o momento onde nos desligamos de tudo aquilo que é demasiadamente estressante e repetitivo em nossa realidade "aparente" e passamos a entrar em contato com a realidade do inconsciente. Desse modo, sonhamos com nossos desejos, nossas paixões, nossas vontades, nossas lembranças mais íntimas e significativas.

Muitas vezes, lembramo-nos de coisas passadas nos nossos sonhos que não lembraríamos com facilidade enquanto acordados. Aquilo que fica guardado em nossa memória, que pensamos ter esquecido ou que não damos tanta atenção, pode ser revelado nos nossos sonhos, a chamada "via régia" para o inconsciente (FREUD, 1899/2010).

Para Freud (1899/2010), os sonhos nos remetem a imagens, fatos, acontecimentos e/ou fantasias da nossa infância. Acontecem de modo brando, mas com alguma ligação com a infância. Porém, em contrapartida, nota-se que os sonhos podem também ocorrer, com fatos que ocorreram há pouco tempo na vida do sonhador e que não são necessariamente, os fatos mais chamativos os marcantes. Isso nos leva a reconhecer o quão impressionante é a nossa memória e que, mesmo aquilo que consideramos sem importância e que pode ser descartado reaparece de algum modo em forma de sonho (FREUD, 1899/2010).

Antigamente, os sonhos eram tidos como "mistérios dos deuses" ou "mensagens divinas" e só aconteceriam em caso de aquiescência divina. Somente quando se começou a estudar os sonhos cientificamente é que pudemos perceber que o mesmo é um retrato da nossa atividade cerebral (FREUD, 1899/2010).

Vários estudos neurofisiológicos sobre os sonhos (DEMENT; KLEITMAN, 1957; DEMENT, 1966/1978; BRYLOWSKI; LEVITAN; LABERGE, 1989; LABERGE, 1985; LABERGE; RHEINGOLD, 1990; LABERGE; GACKENBACH, 2013) evidenciaram a possibilidade de sonhar com estímulos aos quais o sonhador foi exposto imediatamente antes de adormecer. $\mathrm{O}$ conteúdo dos sonhos sofre influência de tais estímulos, ainda que, eventualmente, esses estímulos não sejam retratados tão especificamente quanto da forma como foram apresentados antes do adormecer (LABERGE, 1985; LABERGE; RHEINGOLD, $1990 ; \quad$ LABERGE; GACKENBACH, 2013). Estudos mais recentes (LABERGE; GACKENBACH, 2013) corroboraram a descrição de Freud (1899/2010) segundo a qual os estímulos 
visuais são os de maior eficácia na incubação de um sonho.

Além dos estímulos apresentados antes do adormecer, a maneira como nos comportamos durante o sono, ou seja, a maneira como nos mexemos na cama ou a posição de dormir, também pode influenciar no conteúdo do sonho e produzir sensações intensas durante o sonhar (DEMENT, 1966/1978). Dessa forma, é possível saber como está o funcionamento dos órgãos internos por meio de sonhos, pois os estímulos viscerais também podem se refletir no conteúdo dos sonhos (FREUD, 1899/2010).

Garfield (1974), LaBerge (1985) e LaBerge e Rheingold (1990) demonstraram que é possível aprender a estimular e controlar seus sonhos. Muitas pessoas podem pensar que controlar seu sonho ou sonhar com aquilo que lhe foi estimulado, ou com aquilo que seu cérebro tinha "deletado" seria impossível. Essa crença equivocada ocorre porque grande parte da nossa população não se interessa pelos seus sonhos ou dizem não sonhar.

No entanto, toda pessoa em perfeita saúde física e psíquica consegue sonhas todas as noites, mas a questão é que muitas não se lembram de ter sonhado ou não se lembram do conteúdo de seus sonhos. Isso ocorre, porque nunca somos estimulados a anotar nossos sonhos ou a conta-los para outra pessoa (LABERGE, 1985; LABERGE;
RHEINGOLD, 1990; LABERGE; GACKENBACH，2013). Quando alguém começa a se interessar mais pelos seus sonhos, é mais provável que consiga lembrarse de ter sonhado todas as noites e consiga lembrar até do conteúdo de seus sonhos, o que facilitará para controlar seus sonhos posteriormente (LABERGE, 1985; LABERGE; RHEINGOLD, 1990; LABERGE; GACKENBACH， 2013). Da mesma maneira que podemos sonhar com aquilo que nos aconteceu durante um dia, podemos lembrar-nos de nossos sonhos acerca de determinado acontecimento após termos despertado.

Desse modo, é necessário "aprender a sonhar" para conseguir controlar seus próprios sonhos posteriormente (LABERGE, 1985; LABERGE; RHEINGOLD, 1990; LABERGE; GACKENBACH, 2013). Portanto, influenciar no conteúdo dos próprios sonhos implica se interessar mais ativamente por eles e melhorar a lembrança sobre eles após acordados no mundo real (FREUD, 1899/2010; LABERGE, 1985; LABERGE; RHEINGOLD, 1990).

No trecho de "A Interpretação dos Sonhos" conhecido como "O Material e as Fontes dos Sonhos”, Freud (1899/2010) faz uma análise de seu próprio sonho a respeito de uma monografia acerca de uma planta. A partir da análise desse sonho, Freud (1899/2010) concluiu que os sonhos estabelecem a relação as experiências mais 
antigas da infância, as experiências passadas mais próximas temporalmente e as experiências mais recentes.

Para Freud (1899/2010) nenhum de nós tem sonhos insignificantes ou tolos. Todos os nossos sonhos resgatam lembranças de alguma parte da nossa vida, principalmente a infância. A maneira como ocorre o sonho, seja ela muito real ou muito fantasiosa, depende da nossa lembrança que será evocada e retratada no sonho.

Desse modo, não há sonhos tolos ou sem sentido algum, uma vez que o conteúdo dos nossos sonhos estaria sempre ligado à evocação de alguma de nossas experiências mais antigas ou mais recentes (FREUD, 1899/2010).

\section{Incubação de sonhos}

\subsection{Incubação de Sonhos na Antiguidade e} entre Povos Tradicionais

Garfield (1974) afirma que durante toda a existência da humanidade, os sonhos despertam curiosidade, interesse e busca de significados. Sempre houve civilizações que buscaram os significados de seus sonhos e que mesmo na antiguidade já os incubavam, mesmo que cada um com sua técnica, como por exemplo, na Grécia.

Ao longo do tempo as técnicas se simplificaram, mas é interessante notar, o quanto os gregos já se ocupavam dos sonhos para ter respostas na vida real. Era uma espécie de ritual, onde todos aqueles pertencentes a essa civilização participavam, a fim de sonhar com um Deus. O Deus trazia aos gregos conforto e respostas, para que pudessem suportas as escolhas dos mesmos sobre a vida de mortais. Assim, se tornava mais fácil entender os objetivos de cada Deus e até mesmo, conseguir prever o futuro. O que acontecia também com os índios norteamericanos, que atribuíam seus sonhos para se comunicarem com mensageiros espirituais e quem conseguisse interpretar tais mensageiros, eram respeitados na civilização (GARFIELD, 1974).

É possível notar, o quanto os gregos e os índios norte-americanos atribuíam seus sonhos a sucessos e a decifrar o que os deuses ou os espíritos queriam ensinar aos mortais. Assim, é notável o quanto incubar sonhos já começou a se tornar importante, e que pode ser aprendido por todo aquele que acredita, persiste e tem um objetivo para que aconteça (GARFIELD, 1974).

Há também uma etnia da Malásia, denominada de Senoi, que utiliza a incubação dos sonhos. Nessa cultura, os Senoi aprendem a incubar os sonhos desde pequenos, com o objetivo de a vida a partir das imagens vistas nos sonhos. Dessa forma, aprendendo a controlar o que se sonha, os Senoi conseguem afastar todos os sonhos ditos como "pesadelo", afastando assim o mal e tirando sempre algo de positivo de seus sonhos (GARFIELD, 1974). 
Os sonhadores iogues trazem os sonhos como uma forma de meditação, uma forma terapêutica. De modo que, consigam ter controle sobre seu próprio corpo e estar conscientes que um sonho é sonho quando ainda se está sonhando (GARFIELD, 1974).

Através da descrição das incubações de sonhos feitas pelos Senoi e pelos iogues citados acima, depreende-se que, ao estar acordado em um sonho, é possível torná-lo quase real, sendo possível controlá-lo e ter o controle sobre si mesmo, mesmo após o despertar. O estudo das técnicas Senoi e iogue de incubação de sonhos revela que uma habilidade indispensável à incubação eficaz de sonhos é conseguir se autoconhecer e se autorrealizar.

\subsection{Incubação de Sonhos e Sonhos Lúcidos}

Um sonho lúcido é um sonho ao qual se está consciente de estar sonhando e se é capaz de fazer com que aconteçam fatos e atos que podem lhe proporcionar grandes sensações enquanto se está acordado no sonho e sentir-se bem disposto na vida real (LABERGE, 1985; LABERGE; RHEINGOLD, 1990; LABERGE; GACKENBACH, 2013).

Sonhar lucidamente não se torna tão difícil, quando se tem uma meta a se cumprir. Após aprender os prazeres de se estar acordado em um sonho, um sonhador consegue ter sensações que jamais teria em vida real e melhora sua autoconfiança
(LABERGE,

1985

LABERGE;

RHEINGOLD, 1990).

Contudo, para que se consiga sonhar lucidamente, é preciso que haja foco e disciplina. Aquele que deseja estar acordado em seu sonho precisa acreditar que o conseguirá para que se concretize (LABERGE, $\quad 1985 ; \quad$ LABERGE; RHEINGOLD, $1990 ; \quad$ LABERGE; GACKENBACH, 2013).

Vários estudos comprovam que sonhos lúcidos podem melhorar a qualidade de vida e o bem-estar de uma pessoa, uma vez que promovem vários benefícios a mesma, sendo: melhoria no crescimento e desenvolvimento pessoal; aumento da autoconfiança; melhoria da saúde mental e física, progressão do autocontrole, dentre outros tantos benefícios (GARFIELD, 1974; LABERGE, $\quad 1985$; LABERGE; RHEINGOLD， 1990; DELANEY， 1998; LABERGE; GACKENBACH, 2013).

Com tanta informação nova sobre um simples sonho, seria comum se alguém se perguntasse como isso tudo pode acontecer enquanto uma pessoa dorme para descansar do estresse do dia-a-dia. A resposta, seria simples e fácil: as pessoas precisam aprender a sonhar, precisam descobrir seu mundo interior, novas sensações e conseguir ter consciência de tudo o que está fazendo e tudo que está acontecendo durante o período de sono. 
Os sonhos ocorrem num curto período de tempo, e não a noite toda, como as pessoas costumam imaginar. Ocorrem no período do sono REM, que é a fase do movimento rápido dos olhos (LABERGE, 1985; LABERGE; RHEINGOLD, 1990; LABERGE; GACKENBACH, 2013). Geralmente, ocorre ao adormecer, cerca de 10 a 30 minutos e volta a ocorrer novamente durante a noite, a cada 60 ou 90 minutos. O sono REM, pode ocorrer dentre 5 vezes por noite, é o período em que se está sonhando. No momento em que não está ocorrendo o sono REM, é o período onde há o sono tranquilo, sereno, o sono NÃO REM (DEMENT, 1966/1978; LABERGE, $\quad 1985$; $\quad$ LABERGE; RHEINGOLD, 1990; LABERGE; GACKENBACH, 2013).

Durante nossos sonhos, nosso cérebro permanece ativo, há uma superposição entre a fantasia e a realidade. Em virtude disso, mesmo que os sonhos sejam fantasiosos e criativos, há sempre uma linha que o liga com a realidade, com as experiências reais já vividas (FREUD, 1899/2010). É importante lembrar que devido ao fato de o sonho ser um fenômeno psíquico de base neurofisiológica cíclica, todas as pessoas sonham, todas as noites, por mais que não consigam se lembrar no dia seguinte (LABERGE, 1985; LABERGE; RHEINGOLD, 1990; LABERGE; GACKENBACH, 2013.

Há muito tempo, os sonhos vêm sendo estudado como um conhecimento de si mesmo (FREUD, 1899/2010; JUNG, 1964/2008; HALL, 1983/1997), mas com a prática dos sonhos lúcidos, esse autoconhecimento pode ser intensificado. Além disso, a prática dos sonhos lúcidos pode melhorar nossa disposição para nosso dia-adia e otimizar a resolução criativa de problemas (GARFIELD, 1974; LABERGE, 1985; LABERGE; RHEINGOLD, 1990; DELANEY, 1998; BARRETT, 2001; HILL, 2004; SCHREDER; ERLACHER, 2007; LABERGE; GAKENBACH, 2013).

\subsection{Incubação de Sonhos e Resolução de Problemas}

Quando uma pessoa aprende a incubar sonhos, sente-se muito orgulhosa disso, até mesmo porque essa habilidade é uma ferramenta útil para a resolução de problemas (LABERGE, 1985 ; LABERGE; RHEINGOLD, 1990; DELANEY, 1998). Existem fatos de grandes nomes da história que tiveram sucesso em suas invenções artísticas ou científicas graças a algum determinado sonho que tiveram e que os ajudou a chegar a resolver criativamente um problema que possuíam (DELANEY, 1998; BARRETT, 2001; HILL, 2004).

Um dos objetivos da incubação de sonhos pode ser durante a prática terapêutica ou para o controle de pesadelos - se a pessoa tem pesadelos recorrentes, ela pode aprender a incubar sonhos para evitá-los (DELANEY, 1998; HILL, 2004). Nesse caso, pode-se 
incubar sonhos lúcidos nos quais, primeiramente, a pessoa deverá reconhecer que está tendo um sonho ruim e tentar interrompê-lo imediatamente e transformar o que for negativo em positivo ou mesmo modificar sua reação aos elementos do pesadelo. Isso evitará que a pessoa acorde indisposta ou cansada (DELANEY, 1998/2000).

Estudos mostram que incubar sonhos pode ajudar a resolver problemas pessoais, como ter ideias para crescer nos negócios (DELANEY, 1998; BARRETT, 2001; HILL, 2004; SCHREDER; ERLACHER, 2007). A incubação dos sonhos traz ideias novas e soluciona algum problema que a pessoa não conseguia enxergar durante seu período da vida em vigília (DELANEY, 1998). Arquitetos e engenheiros podem usar os sonhos para estimular a criação de formas e desenhos que possam ser a peça chave de um projeto. É possível dizer, que os sonhos podem influenciar em todas as culturas e todas as profissões que demandam soluções criativas e/ou inovadoras (BARRETT, 2001; SCHREDER; ERLACHER, 2007).

Os sonhos beneficiam também na saúde de quem o incuba, pois o sonhador consegue se conhecer como um todo e então podem perceber ao analisar determinado sonho, que alguma parte de seu corpo precisa de cuidados, antecipando o tratamento e no caso de uma doença grave, diminuindo também a culpa e os riscos de vida do paciente (DELANEY, 1998; HILL, 2004).

\section{Principais técnicas de incubação de sonhos}

Muitas pessoas podem achar inicialmente, a incubação de sonhos algo estranho e sem nexo, isso porque nunca fomos realmente apresentados aos nossos sonhos, ou seja, nunca nos ensinaram o quanto os sonhos podem nos beneficiar, nos melhorar e consequentemente, melhorar a sociedade em que vivemos (DELANEY, 1998).

Para incubar sonhos, é necessário concentração, foco e disciplina (LABERGE, 1985; LABERGE; RHEINGOLD, 1990; DELANEY, 1998). Prestar atenção ao sonho e relatá-lo depois de acordar é uma técnica preliminar essencial para ajudar a melhorar a incubação dos sonhos.

Algumas regras básicas são: fazer um diário dos seus sonhos, ou seja, anotar todos os dias tudo aquilo que você sonhou procurar um lugar em que consiga se relaxar por inteiro, pensar fixamente naquilo que se deseja sonhar, para adormecer com tal desejo na mente.

É claro que criar um ritual antes de dormir não é fácil, mas para quem deseja "sonhar melhor", quem deseja se encontrar em seus sonhos, é preciso um grande esforço e concentração, pois só assim conseguirá incubar sonhos. 
O primeiro passo para treinar a incubação de sonhos é estar descansado e não fazer o uso de medicação e/ou entorpecentes para adormecer. Depois, antes de adormecer, é importante que faça anotações sobre como foi o seu dia. Quando despertar, deve-se falar sobre o tema incubado e discutir/refletir sobre o problema, lembrando que esse processo só deve acontecer quando o sonhador sentir-se preparado.

É importante que seja anotada uma frase antes de dormir com o objetivo de obterse a incubação, com objetivos claros, para facilitar o sonho a ser incubado. A frase deve ser sempre a mesma e deve ser repetida continuamente e concentradamente até adormecer. Então, basta adormecer serena e tranquilamente e sonhar.

Sempre que acordar, o sonho deve ser anotado com o máximo de lembrança possível e na noite seguinte, tentar repetir o sonho. Depois, é importante fazer entrevistas com você mesmo, a respeito de seus sonhos. Não se devem forçar seus sonhos a lhe dar respostas, ele deve ser explorado sempre com a mente aberta.

Há vários tipos de técnicas para incubar um sonho. A técnica MILD trata especificamente de dormir pensando em acordar cedo e após acordar, realizar 15 minutos de alguma atividade e então voltar para a cama e dormir novamente, voltando para o sonho.
O Método de Tholey consiste em se perguntar se está realmente sonhando ou não e tentar realizar uma ação durante o sonho para que o fato seja constatado.

Já a Indução Hipnótica se resume ao sonho ser induzido após a pessoa adormecer e a mesma terá consciência de que está sonhando. É como ter um acompanhante, ou ter um ritual ao adormecer.

Há também o método RISC, que consiste basicamente em rever os sonhos incubados e, sempre que o sonho for ruim, tentar imaginar finais diferentes para ele. Há ainda o método em que se tenta imaginar imagens diferentes, não importa o que aconteça no sonho; registrar um pesadelo após acordar, a fim de eliminá-lo; e, mudar seu pesadelo ruim, para um sonho bom. Após um pesadelo, imagine sonhos bons e incubeos.

\section{Conclusão}

O objetivo do presente estudo foi descrever o panorama sobre as teorias e as técnicas relativas ao tema da incubação de sonhos. As teorias explicativas mais relevantes e as técnicas principais para a realização da incubação de sonhos foram descritas e discutidas, permitindo a elaboração de um panorama sintético a respeito da incubação de sonhos.

\section{Referências}

BARRETT, D. The committee of sleep:

How artists, scientists, and athletes use 
dreams for creative problem-solving - and how you can too. New York: Crown, 2001.

BRYLOWSKI, A.; LEVITAN, L.;

LABERGE, S. H-reflex supression and autonomic activation during lucid REM sleep: a case study. Sleep, 12, p.374-378.

\section{DELANEY, G. O Livro de Ouro dos}

Sonhos. Rio de janeiro: Ediouro, 1998.

DEMENT, W.; KLEITMAN, N. Cyclic variations in EEG during sleep and their relation to eye movements, body motility and dreaming. Electroencephalography and Clinical Neurophysiology, 9, p.673-690.

DEMENT, W. A Psicofisiologia do Sonho. In: CAILLOIS, Roger; GRUNEBAUN, G. E. Von. O Sonho e as Sociedades Humanas. Rio de Janeiro: Editora Francisco Alves, 1966/1978.

FREUD, S. A Interpretação dos Sonhos. Vol. I. Obras Completas v. IV. São Paulo: Cia das Letras, 1899/2010.

GARFIELD, P. Sonhos Criativos - aprenda a controlar e programar seus sonhos. Rio de Janeiro: Nova Fronteira, 1974.

HALL, J. A. Jung e a Interpretação dos Sonhos. São Paulo: Editora Cultrix, 1997.

HILL, C. E. Dream work in therapy: Facilitating exploration, insight, and action. Washington, DC: American Psychological Association, 2004.

JUNG, C. G. Símbolos e Interpretação dos Sonhos. In - A Vida Simbólica. Vol. I. Obras Completas vol. XVIII. Petrópolis: Editora Vozes, 1964/2008a.

JUNG, C. G. O homem e seus símbolos. Petrópolis: Editora Vozes, 1964/2008b.

LABERGE, S. Sonhos Lúcidos. São Paulo:

Editora Siciliana, 1985.
LABERGE, S.; RHEINGOLD, H. Exploring the world of lucid dreaming. New York: Balantine, 1990.

LABERGE, S.; GACKENBACH, J. O sonhar lúcido. In: E. Cardeña; S.J. Lynn; S. Krippner (Orgs). Variedades da experiência anômala: análise de evidências científicas. São Paulo: Atheneu, 2013.

SCHREDL, M.; ERLACHER, D. SelfReported Effects of Dreams on Waking-Life Creativity: An Empirical Study The Journal of Psychology, 141(1), p.35-46, 2007.

WHITE, G.L.; TAYTROE, L. Personal Problem-Solving Using Dream Incubation: Dreaming, Relaxation, or Waking Cognition? Dreaming, 13(4), p.193-209, 2003. 